\title{
The pseudo-gap phase and the duality in holographic fermionic system with dipole coupling on Q-lattice
}

\author{
Yi Ling ${ }^{1,3}$, * Peng Liu ${ }^{1}$ 甼 Chao Niu ${ }^{1}$ 用 and Jian-Pin $\mathrm{Wu}^{2,3 \S}$ \\ 1 Institute of High Energy Physics, \\ Chinese Academy of Sciences, Beijing 100049, China \\ ${ }^{2}$ Institute of Gravitation and Cosmology, \\ Department of Physics, School of Mathematics and Physics, \\ Bohai University, Jinzhou 121013, China \\ 3 State Key Laboratory of Theoretical Physics, Institute of Theoretical Physics, \\ Chinese Academy of Sciences, Beijing 100190, China
}

\begin{abstract}
We classify the different phases by the "pole-zero mechanism" for a holographic fermionic system which contains a dipole coupling with strength $p$ on a Q-lattice background. A complete phase structure in $p$ space can be depicted in terms of Fermi liquid, non-Fermi liquid, Mott phase and pseudo-gap phase. In particular, we find that in general the region of the pseudo-gap phase in $p$ space is suppressed when the Q-lattice background is dual to a deep insulating phase, while for an anisotropic background, we have an anisotropic region for the pseudo-gap phase in $p$ space as well. In addition, we find that the duality between zeros and poles always exists regardless of whether or not the model is isotropic.
\end{abstract}

Key Words: Holographic Q-lattice, "pole-zero mechanism”, Fermionic system

PACS: $11.25 . \mathrm{Tq}, 04.70 . \mathrm{Bw}$

*Electronic address: lingy@ihep.ac.cn

${ }^{\dagger}$ Electronic address: liup51@ihep.ac.cn

‡Electronic address: niuc@ihep.ac.cn

$\S$ Electronic address: jianpinwu@mail.bnu.edu.cn 


\section{INTRODUCTION}

Until now, a general theoretical framework for quantum phases and phase transitions of strongly correlated electron systems, such as cuprate and other oxides, has not been established yet. As an alternative approach, AdS/CFT correspondence [1 3] is a powerful tool to attack these strongly correlated problems and to get some possible clues on the basic principle behind these numerous correlated electron systems.

Indeed, some exotic states of matter, including Fermi liquid, non-Fermi liquid, Mott phase and pseudo-gap phase, have been found or identified by AdS/CFT correspondence [4-11]. By adding a probe fermion on RN-AdS background, a non-linear dispersion relation is observed [5], indicating that a non-Fermi liquid phase emerges. Also, it is found that the low energy behavior of a fermionic system on the RN-AdS background is controlled by the $A d S_{2}$ near horizon geometry [6]. Later, the properties of a fermionic system on Gauss-Bonnet, Lifshitz as well as hyperscaling violation geometry have been extensively studied [12 21]. Furthermore, a dipole coupling between the gauge field and the Dirac field can be introduced to model the Mott phase [8, 9]. Many extended works on the dipole coupling effects have been explored in more general geometries in [22 28]. Recently, in terms of the "pole-zero mechanism", a pseudo-gap phase has been detected in a holographic fermionic system with dipole coupling in RN-AdS black hole and the Schwarzschild-AdS black hole [10, 11]. Moreover, they observed a remarkable duality between zeroes and poles in this holographic system [10], which should be interesting for experimental scientists in condensed matter physics, although this phenomenon has not been captured by experiments yet. In this paper, we shall address the pseudo-gap phase and the duality in a holographic fermionic system with dipole coupling on Q-lattice geometry.

Motivated by the idea of Q-balls [29], a Q-lattice model in a holographic framework was first constructed in [30], in which the translational symmetry was broken and a metalinsulator transition observed through the study of optical conductivity. A lot of relevant work has been investigated in this context [31 37]. In [35], we study a holographic fermionic system with dipole coupling on Q-lattice geometry and find that a Mott gap opens when the dipole coupling parameter $p$ is beyond a certain critical value $p_{c}$. An interesting result is that the Mott gap opens much more easily when the Q-lattice background is dual to a deep insulating phase rather than a metallic phase. Here, we shall probe the pseudo-gap phase in 
this system by the "pole-zero mechanism" and study how the lattice parameters affect the formation of the pseudo-gap.

Our paper is organized as follows. In Section II, a brief review of the holographic Qlattice geometry and the Dirac equation is presented. Our main results on the classification of quantum phases of holographic fermionic system with dipole coupling are presented for isotropic and anisotropic Q-lattice geometry in Section III and Section IV] respectively. Specifically, we will focus on the pseudo-gap phase and discuss how the lattice parameters affect its formation. Finally, the conclusion and discussion are presented in Section $\mathrm{V}$.

\section{HOLOGRAPHIC Q-LATTICE GEOMETRY AND THE DIRAC EQUATION}

In this section, we give a brief introduction on the holographic Q-lattice model which breaks translational symmetry in both spatial directions and then demonstrate how to simplify the Dirac equation over a specific Q-lattice background. For detailed discussion, we refer to [30, 34, 35].

The action with Q-lattice structure in both of the spatial directions can be written as

$$
S=\int d^{4} x \sqrt{-g}\left[R+6-\frac{1}{2} F^{2}-\left|\partial \phi_{1}\right|^{2}-m_{1}^{2}\left|\phi_{1}\right|^{2}-\left|\partial \phi_{2}\right|^{2}-m_{2}^{2}\left|\phi_{2}\right|^{2}\right],
$$

where $F=d A$. $\phi_{1}$ and $\phi_{2}$ are the complex scalar fields simulating the lattices. From the above action, the equations of motion can be deduced as

$$
\begin{aligned}
& R_{\mu \nu}=g_{\mu \nu}\left(-3+\frac{m_{1}^{2}}{2}\left|\phi_{1}\right|^{2}+\frac{m_{2}^{2}}{2}\left|\phi_{2}\right|^{2}\right)+\left(F_{\mu}{ }^{\rho} F_{\nu \rho}-\frac{1}{4} g_{\mu \nu} F^{2}\right)+\partial_{(\mu} \phi_{1} \partial_{\nu)} \phi_{1}^{*}+\partial_{(\mu} \phi_{2} \partial_{\nu)} \phi_{2}^{*} \\
& \left(\nabla^{2}-m_{1}^{2}\right) \phi_{1}=0, \quad\left(\nabla^{2}-m_{2}^{2}\right) \phi_{2}=0, \quad \nabla_{\mu} F^{\mu \nu}=0
\end{aligned}
$$

To find solutions to the above equations of motion, we take the following ansatz

$$
\begin{aligned}
& d s^{2}=-g_{t t}(z) d t^{2}+g_{z z}(z) d z^{2}+g_{x x}(z) d x^{2}+g_{y y}(z) d y^{2}, \\
& \phi_{1}=e^{i k_{1} x} \varphi_{1}, \quad \phi_{2}=e^{i k_{2} y} \varphi_{2}, \quad A=A_{t}(z) d t
\end{aligned}
$$

with

$$
\begin{aligned}
& g_{t t}(z)=\frac{(1-z) P(z) Q(z)}{z^{2}}, \quad g_{z z}(z)=\frac{1}{z^{2}(1-z) P(z) Q(z)}, \\
& g_{x x}(z)=\frac{V_{1}(z)}{z^{2}}, \quad g_{y y}(z)=\frac{V_{2}(z)}{z^{2}}, \quad A_{t}(z)=\mu(1-z) a(z), \\
& P(z)=1+z+z^{2}-\frac{\mu^{2} z^{3}}{2},
\end{aligned}
$$


where $k_{1}$ and $k_{2}$ are two wave-numbers along $x$ and $y$ directions, respectively, such that the $\phi_{1,2}$ is periodic in $x, y$ direction with lattice constant $2 \pi / k_{1,2}$. In addition, in this paper, we will set $m_{1,2}^{2}=-5 / 4$ for definiteness, which avoids the violation of the $A d S_{2}$ BF bound near the horizon.

Based on the above ansatz, we obtain five second order ODEs for $V_{1}, V_{2}, a, \varphi_{1}, \varphi_{2}$ and one first order ODE for $Q$. To solve the ODEs numerically, we impose a regular boundary condition at the horizon $z=1$ and impose the following conditions on the conformal boundary:

$$
Q(0)=1, \quad V_{1}(0)=V_{2}(0)=1, \quad a(0)=1 .
$$

We will only focus on the standard quantisation of the scalar field where the asymptotic behavior of $\varphi_{1,2}$ is described as $\varphi_{1,2}=\lambda_{1,2} z^{\Delta_{1-, 2-}}$, with the UV behavior corresponding to Q-lattice deformation with lattice amplitude $\lambda_{1,2}$, where $\Delta_{1 \pm, 2 \pm}=3 / 2 \pm\left(9 / 4+m_{1,2}^{2}\right)^{1 / 2}$, is the scaling dimension of the dual field of $\phi_{1,2}$. In addition, the Hawking temperature of the black hole is given by

$$
T=\frac{P(1) Q(1)}{4 \pi} .
$$

As a result, each of our Q-lattice solutions can be specified by five dimensionless quantities $T / \mu, \lambda_{1} / \mu^{\Delta_{1-}}, \lambda_{2} / \mu^{\Delta_{2-}}, k_{1} / \mu$ and $k_{2} / \mu$. We shall abbreviate these quantities to $T, \lambda_{1,2}, k_{1,2}$ respectively in what follows for simpleness.

Now, we introduce the Dirac equation with dipole coupling on this Q-lattice geometry. We consider the following action, which involves the interaction between the spinor field and the gauge field

$$
S_{D}=i \int d^{4} x \sqrt{-g} \bar{\zeta}\left(\Gamma^{a} \mathcal{D}_{a}-m_{\zeta}-i p \not{F}\right) \zeta
$$

In the above action, $\mathcal{D}_{a}=\partial_{a}+\frac{1}{4}\left(\omega_{\mu \nu}\right)_{a} \Gamma^{\mu \nu}-i q A_{a}$ and $\not{F}=\frac{1}{2} \Gamma^{\mu \nu}\left(e_{\mu}\right)^{a}\left(e_{\nu}\right)^{b} F_{a b}$, where $\left(e_{\mu}\right)^{a}$ form a set of orthogonal normal vector bases and $\left(\omega_{\mu \nu}\right)_{a}$ is the spin connection 1-forms. With the redefinition $\zeta=\left(g_{t t} g_{x x} g_{y y}\right)^{-1 / 4} \mathcal{F}$, and by denoting the Fourier transform of $\mathcal{F}$ as $F(z, \mathbf{k}) \equiv\left(\mathcal{A}_{\alpha}, \mathcal{B}_{\alpha}\right)^{T}$ with $\alpha=1,2$, and choosing the following gamma matrices,

$$
\Gamma^{3}=\left(\begin{array}{cc}
-\sigma^{3} & 0 \\
0 & -\sigma^{3}
\end{array}\right), \quad \Gamma^{0}=\left(\begin{array}{cc}
i \sigma^{1} & 0 \\
0 & i \sigma^{1}
\end{array}\right),
$$




$$
\Gamma^{1}=\left(\begin{array}{cc}
-\sigma^{2} & 0 \\
0 & \sigma^{2}
\end{array}\right), \quad \Gamma^{2}=\left(\begin{array}{cc}
0 & \sigma^{2} \\
\sigma^{2} & 0
\end{array}\right)
$$

the Dirac equation deduced from Eq.(7) can be written in a simple form as

$$
\begin{aligned}
& \left(\frac{1}{\sqrt{g_{z z}}} \partial_{z} \mp m_{\zeta}\right)\left(\begin{array}{l}
\mathcal{A}_{1} \\
\mathcal{B}_{1}
\end{array}\right) \pm\left(\omega+q A_{t}\right) \frac{1}{\sqrt{g_{t t}}}\left(\begin{array}{l}
\mathcal{B}_{1} \\
\mathcal{A}_{1}
\end{array}\right)+\frac{p}{\sqrt{g_{z z} g_{t t}}}\left(\partial_{z} A_{t}\right)\left(\begin{array}{l}
\mathcal{B}_{1} \\
\mathcal{A}_{1}
\end{array}\right) \\
& -\frac{k_{x}}{\sqrt{g_{x x}}}\left(\begin{array}{l}
\mathcal{B}_{1} \\
\mathcal{A}_{1}
\end{array}\right)+\frac{k_{y}}{\sqrt{g_{y y}}}\left(\begin{array}{l}
\mathcal{B}_{2} \\
\mathcal{A}_{2}
\end{array}\right)=0, \\
& \left(\begin{array}{l}
\frac{1}{\sqrt{g_{z z}}} \partial_{z} \mp m_{\zeta}
\end{array}\right)\left(\begin{array}{l}
\mathcal{A}_{2} \\
\mathcal{B}_{2}
\end{array}\right) \pm\left(\omega+q A_{t}\right) \frac{1}{\sqrt{g_{t t}}}\left(\begin{array}{l}
\mathcal{B}_{2} \\
\mathcal{A}_{2}
\end{array}\right)+\frac{p}{\sqrt{g_{z z} g_{t t}}}\left(\partial_{z} A_{t}\right)\left(\begin{array}{l}
\mathcal{B}_{2} \\
\mathcal{A}_{2}
\end{array}\right) \\
& +\frac{k_{x}}{\sqrt{g_{x x}}}\left(\begin{array}{l}
\mathcal{B}_{2} \\
\mathcal{A}_{2}
\end{array}\right)+\frac{k_{y}}{\sqrt{g_{y y}}}\left(\begin{array}{l}
\mathcal{B}_{1} \\
\mathcal{A}_{1}
\end{array}\right)=0 .
\end{aligned}
$$

To solve the Dirac equation, we need to impose the following independent ingoing boundary condition at the horizon

$$
\left(\begin{array}{c}
\mathcal{A}_{\alpha}(z, \mathbf{k}) \\
\mathcal{B}_{\alpha}(z, \mathbf{k})
\end{array}\right)=c_{\alpha}\left(\begin{array}{c}
1 \\
-i
\end{array}\right)(1-z)^{-\frac{i \omega}{4 \pi T}}
$$

The near boundary behavior of the Dirac field will be

$$
\left(\begin{array}{l}
\mathcal{A}_{\alpha} \\
\mathcal{B}_{\alpha}
\end{array}\right) \approx a_{\alpha} z^{m_{\zeta}}\left(\begin{array}{l}
1 \\
0
\end{array}\right)+b_{\alpha} z^{-m_{\zeta}}\left(\begin{array}{l}
0 \\
1
\end{array}\right)
$$

Finally, we can read off the retarded Green function by holography

$$
a_{\alpha}=G_{\alpha \alpha^{\prime}} b_{\alpha^{\prime}}
$$

To obtain the boundary Green function, we need to construct a basis of finite solutions, $\left(\mathcal{A}_{\alpha}^{I}, \mathcal{B}_{\alpha}^{I}\right)$ and $\left(\mathcal{A}_{\alpha}^{I I}, \mathcal{B}_{\alpha}^{I I}\right)$ due to the four components of the Dirac fields being coupled to one another.

\section{PSEUDO-GAP PHASES AND DUALITY ON AN ISOTROPIC Q-LATTICE}

There is competition between the poles $\left(k=k_{F}\right)$ and zeros $\left(k=k_{L}\right)$ in the Green function

of a strongly coupled fermionic system in condensed matter physics. By the "pole-zero 
mechanism", we can classify the different phases of a strongly coupled fermionic system [3842]. The criterion of the phase classification is given as below.

$$
\begin{aligned}
& \text { Poles } \Leftrightarrow \text { (Non-)Fermi liquid phase } \\
& \text { Zeros } \Leftrightarrow \quad \text { Mott Insulator phase } \\
& \text { Coexistence of poles and zeros } \Leftrightarrow \quad \text { Pseudo-gap phase }
\end{aligned}
$$

In a holographic framework, this "pole-zero mechanism" was first introduced in [10] to characterize different phases. Here, we will use this mechanism to probe the pseudo-gap phase in the holographic fermionic system with dipole coupling on Q-lattice geometry. We explore the case of an isotropic Q-lattice in this section, i.e., $\lambda_{1}=\lambda_{2}$ and $k_{1}=k_{2}$, in which we can set $k_{y}=0$ without loss of generality. The case of anisotropic Q-lattice geometry will be discussed in the next section.

For definiteness, we fix $m=0$ as well as $q=1$ and work at an extremely low temperature $T \simeq 0.00398$ throughout this paper.

Firstly, for $p=0$, neither poles nor zeros can be observed in the determinant of the Green function $\operatorname{det} G_{R}$ because the poles (zeros) of Green function are cancelled (Fig. 1). However, once the dipole coupling is turned on, the poles or zeros emerge in $\operatorname{det} G_{R}$ so that we can classify the phases for the fermionic system with dipole coupling in terms of the "pole-zero mechanism". Fig. 2 shows the determinant of the Green function $\operatorname{det} G_{R}$ as a function of $k_{x}$ at $\omega=0$ for $p=-4.5$ (Fig. 1(a) and $p=4.5$ (Fig. 1(b) with $\lambda_{1}=\lambda_{2}=0.5$ and $k_{1}=k_{2}=0.8$. One pole emerges at $k_{x}=k_{F} \simeq 1.222$ for $p=-4.5$, indicating a (non-)Fermi liquid state, and a zero can be found at the same momentum $k_{x}=k_{L} \simeq 1.222$ for $p=4.5$, indicating a Mott state. Obviously, there is a duality between zero and pole under $p \rightarrow-p$, which was first revealed in [10. When we decrease the dipole coupling to $|p|=0.1$, we find the coexistence of pole and zero in $\operatorname{det} G_{R}$ (Fig. 3), which points to a pseudo-gap phase. Again, the duality between zeros and poles remains under $p \rightarrow-p$ (Fig. 3). According to the above observations, we claim that the (non-)Fermi liquid phase, Mott phase and the pseudo-gap phase emerge in a fermionic system with dipole coupling on the Q-lattice. By the density of state (DOS) $A(\omega)$, we can determine the critical point $p_{c}$ of the transition from (non-)Fermi liquid phase to Mott phase and find that the Mott gap opens more easily in a deep insulating phase than a metallic phase [35]. Now, we shall focus on the effect of lattice constant $k_{1,2}$ and lattice amplitude $\lambda_{1,2}$ on the pseudo-gap phase. 


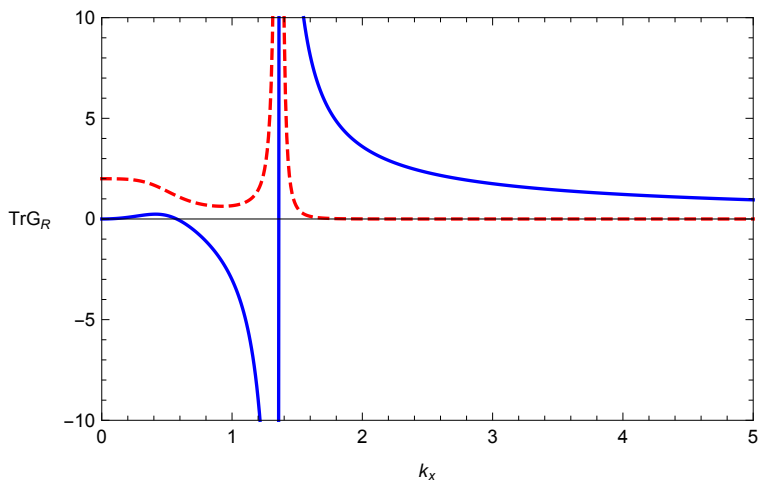

(a)

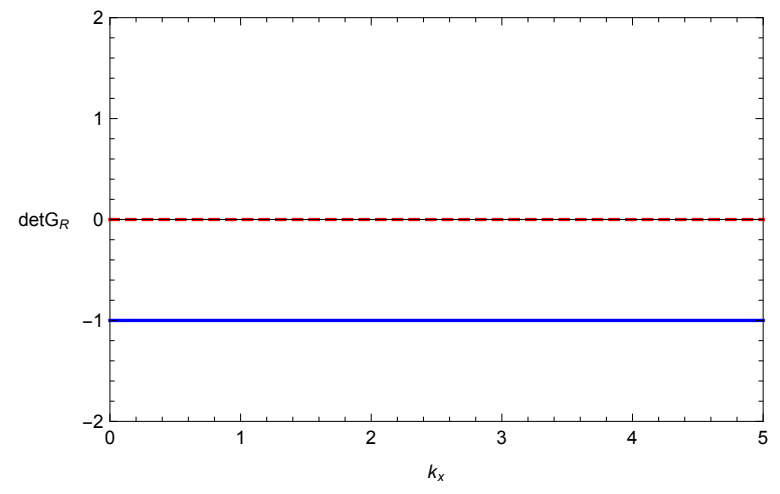

(b)

FIG. 1: (a): $\operatorname{Re}\left(\operatorname{Tr} G_{R}\right)$ (solid blue line) and $\operatorname{Im}\left(\operatorname{Tr} G_{R}\right)$ (dashed red line) for $p=0$ at $\omega=0$. A pole is visible in the spectral function $A\left(k_{x}\right)\left(\operatorname{Im}\left(\operatorname{Tr} G_{R}\right)\right)$, which indicates a Fermi surface $\left(k_{F} \simeq 1.359\right)$. (b): $\operatorname{Re}\left(\operatorname{det} G_{R}\right)$ (solid blue line) and $\operatorname{Im}\left(\operatorname{det} G_{R}\right)$ (dashed red line) for $p=0$ at $\omega=0$, in which neither poles nor zeroes is observed. Here, we have fixed $\lambda_{1}=\lambda_{2}=0.5$ and $k_{1}=k_{2}=0.8$.

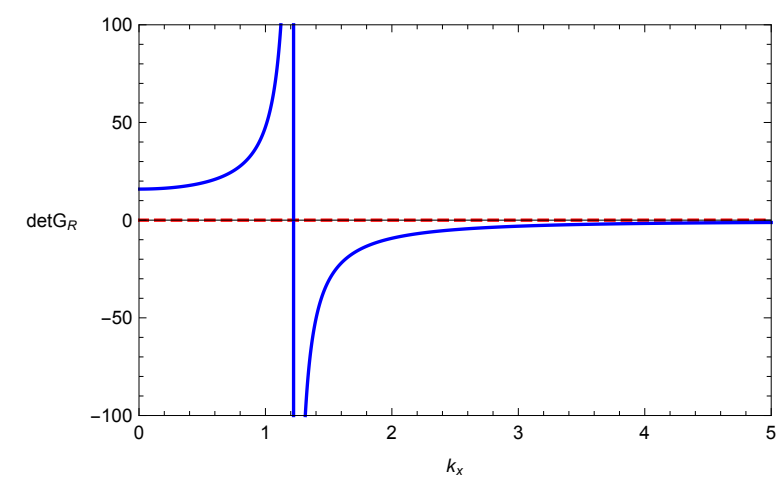

(a)

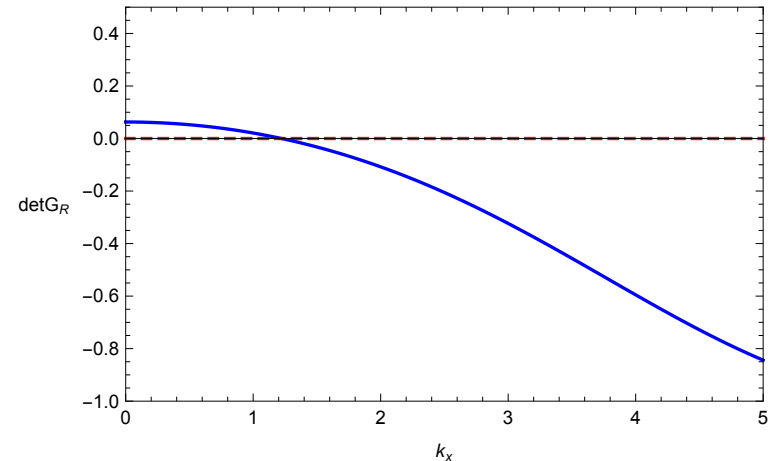

(b)

FIG. 2: $\operatorname{Re}\left(\operatorname{det} G_{R}\right)$ (solid blue line) and $\operatorname{Im}\left(\operatorname{det} G_{R}\right)$ (dashed red line) at $\omega=0$. (a) $(p=-4.5)$ shows a pole at $k_{x}=k_{F} \simeq 1.222$ and (b) $(p=4.5)$ shows a zero at $k_{x}=k_{L} \simeq 1.222$. Here, we have fixed $\lambda_{1}=\lambda_{2}=0.5$ and $k_{1}=k_{2}=0.8$.

By careful numerical calculation, we find that a pseudo-gap emerges when $|p| \lesssim 0.605$ for $\lambda_{1}=\lambda_{2}=0.5$ and $k_{1}=k_{2}=0.8$. When we go to a deep insulating phase with $\lambda_{1}=\lambda_{2}=2$ and $k_{1}=k_{2}=1 / 2^{3 / 2}$, the pseudo-gap phase emerges in the region of $|p| \lesssim 0.335$. Therefore, the region of the pseudo-gap phase in $p$ space is suppressed in the deep insulating phase. For comparison, we also find that the pseudo-gap phase appears when $|p| \lesssim 0.634$ in the 


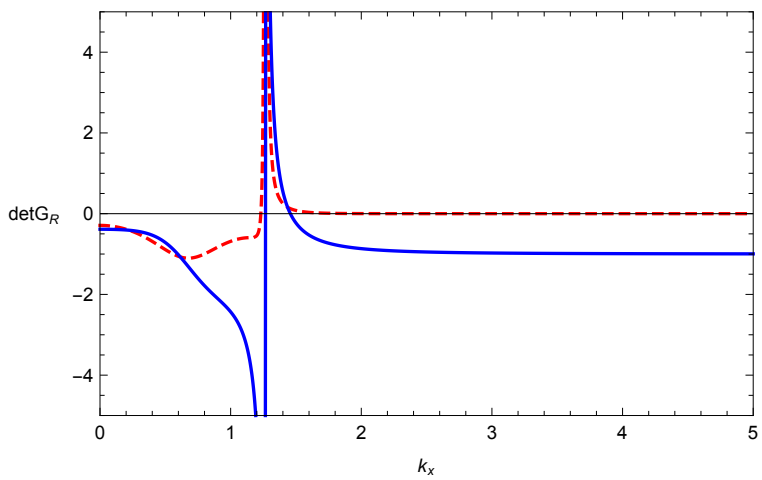

(a)

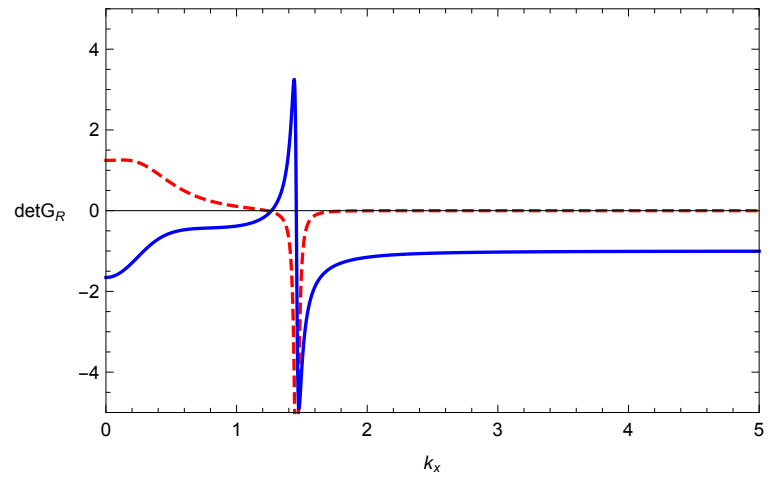

(b)

FIG. 3: $\operatorname{Re}\left(\operatorname{det} G_{R}\right)$ (solid blue line) and $\operatorname{Im}\left(\operatorname{det} G_{R}\right)$ (dashed red line) at $\omega=0$. Both pole and zero can be seen for $p=-0.1\left((\mathrm{a}): k_{x}=k_{L} \simeq 1.441\right.$ and $\left.k_{x}=k_{F} \simeq 1.274\right)$ and $p=0.1((\mathrm{~b})$ : $k_{x}=k_{L} \simeq 1.274$ and $\left.k_{x}=k_{F} \simeq 1.441\right)$. Here, we have fixed $\lambda_{1}=\lambda_{2}=0.5$ and $k_{1}=k_{2}=0.8$.

RN-AdS black hole background, which is obtained simply by setting $\lambda_{1}=\lambda_{2}=0^{1}$.

Before closing this section, we would like to present a brief discussion on the duality between zeros and poles. When $k_{y}$ is set to zero, we can package the Dirac equations (9) and (10) into the following evolution equations

$$
\left(\partial_{z}-2 m_{\zeta} \sqrt{g_{z z}}\right) \xi_{\alpha}+\left[v_{-}+(-1)^{\alpha} k \sqrt{\frac{g_{z z}}{g_{x x}}}\right]+\left[v_{+}-(-1)^{\alpha} k \sqrt{\frac{g_{z z}}{g_{x x}}}\right] \xi_{\alpha}^{2}=0,
$$

where we have defined $\xi_{\alpha} \equiv \frac{\mathcal{A}_{\alpha}}{\mathcal{B}_{\alpha}}$ and $v_{ \pm}=\sqrt{\frac{g_{z z}}{g_{t t}}}\left(\omega+q A_{t}\right) \pm p \sqrt{g^{t t}} \partial_{z} A_{t}$. For massless fermions, the retarder Green function is

$$
G(\omega, k)=\lim _{z \rightarrow 0}\left(\begin{array}{cc}
\xi_{1} & 0 \\
0 & \xi_{2}
\end{array}\right)
$$

Therefore, from the evolution equation (14), one can easily find the following symmetry of Green's function

$$
G_{11}(\omega, k)=G_{22}(\omega,-k)
$$

In addition, we can introduce the reciprocal of $\xi_{\alpha}$,

$$
\oiint_{\alpha}=\frac{1}{\xi_{\alpha}} .
$$

\footnotetext{
${ }^{1}$ Because we have set the gauge coupling constant $g_{F}=\sqrt{2}$ here, which is different from the conventions in [10] that $g_{F}=2$, the charge $q$ and dipole coupling $p$ will correspond to $\sqrt{2} q$ and $\sqrt{2} p$ in [10] due to the products $g_{F} q$ and $g_{F} p$ being the relevant quantities.
} 
We find that $\oiint_{\alpha}$ satisfies the following evolution equations

$$
\left(\partial_{z}-2 m_{\zeta} \sqrt{g_{z z}}\right) \oiint_{\alpha}+\left[\psi_{-}+(-1)^{\alpha} k \sqrt{\frac{g_{z z}}{g_{x x}}}\right]+\left[\psi_{+}-(-1)^{\alpha} k \sqrt{\frac{g_{z z}}{g_{x x}}}\right] \dot{\xi}_{\alpha}^{2}=0,
$$

where $\psi_{ \pm}=-\sqrt{\frac{g_{z z}}{g_{t t}}}\left(\omega+q A_{t}\right) \pm p \sqrt{g^{t t}} \partial_{z} A_{t}$. It is easily found that there is a symmetry between Eqs. (14) and (18) under the transformation $k \rightarrow-k, p \rightarrow-p$ and $\xi_{\alpha} \rightarrow-\oiint_{\alpha}$. Combining Eq. (16), we can easily find that there is a duality between zeros and poles when $p \rightarrow-p$.

\section{PSEUDO-GAP PHASES AND DUALITY ON AN ANISOTROPIC Q- LATTICE}

Now we turn to study the pseudo-gap phase and the duality between the zeros and poles on anisotropic Q-lattices, in which we set $\lambda_{1}=1, \lambda_{2}=0.1$ and $k_{1}=k_{2}=0.8$.

Firstly, we explore the duality along the $k_{x}$ direction (setting $k_{y}=0$ ). As illustrated in Fig. 4 and Fig. 5, we find that the (non-)Fermi liquid phase, Mott insulating phase and the pseudo-gap phase along the $k_{x}$ direction emerge, depending on the values of the dipole coupling parameter $p$. Furthermore, the duality between zeros and poles still holds, which can also be understood by a similar analysis to the last section. In a parallel manner, we also work out the location of poles and zeros in $\operatorname{det} G_{R}$ along the $k_{y}$ direction (setting $k_{x}=0$ ) for $p=6$ and $p=-6$. We find that $k_{y F} \simeq 1.983$ for $p=-6$ and $k_{y L} \simeq 1.983$ for $p=6$, which indicates that the duality remains valid in the $y$ direction. But obviously we notice that $k_{x F} \neq k_{y F}\left(k_{x L} \neq k_{y L}\right)$, reflecting the anisotropy of the system.

We now study the pseudo-gap phase. We find that the pseudo-gap phase emerges along the $k_{x}$ direction for $|p| \lesssim 0.393$, while along the $k_{y}$ direction for $|p| \lesssim 0.860$. Obviously,

the anisotropic geometry results in the anisotropic pseudo-gap phase region. Along the insulating direction ( $k_{x}$ direction), the region of pseudo-gap phase is suppressed, which is consistent with that explored on isotropic Q-lattices in the previous section.

\section{CONCLUSION AND DISCUSSION}

By the "pole-zero mechanism" we have classified the phases which appear in a holographic fermionic system with dipole coupling on Q-lattice geometry. These phases could be Fermi liquid, non-Fermi liquid, Mott phase or pseudo-gap phase, depending on the strength of the 


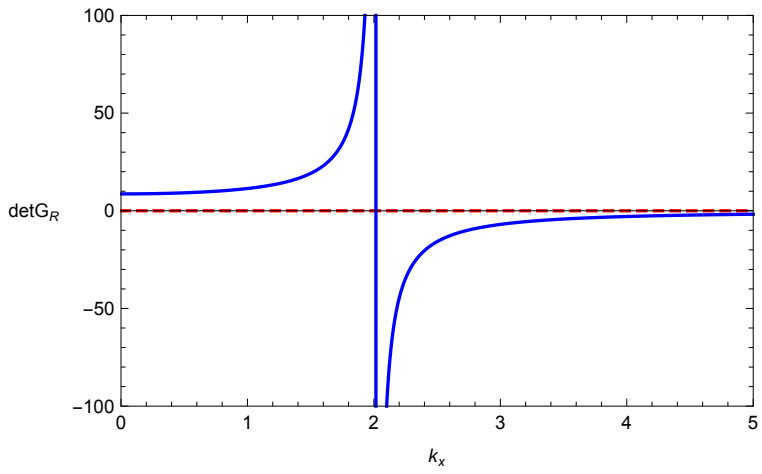

(a)

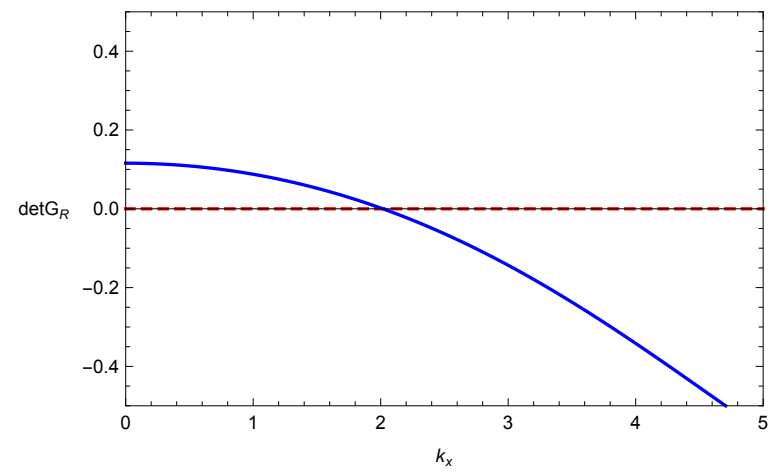

(b)

FIG. 4: $\operatorname{Re}\left(\operatorname{det} G_{R}\right)$ (solid blue line) and $\operatorname{Im}\left(\operatorname{det} G_{R}\right)$ (dashed red line) as a function of $k_{x}$ (we set $\left.k_{y}=0\right)$ at $\omega=0$ with $p=-6((\mathrm{a}))$ and $p=6((\mathrm{~b}))$ on anisotropic Q-lattice background with $\lambda_{1}=1, \lambda_{2}=0.1$ and $k_{1}=k_{2}=0.8$. (a) shows a pole at $k_{x}=k_{x F} \simeq 2.016$ and (b) shows a zero at $k_{x}=k_{x L} \simeq 2.016$.

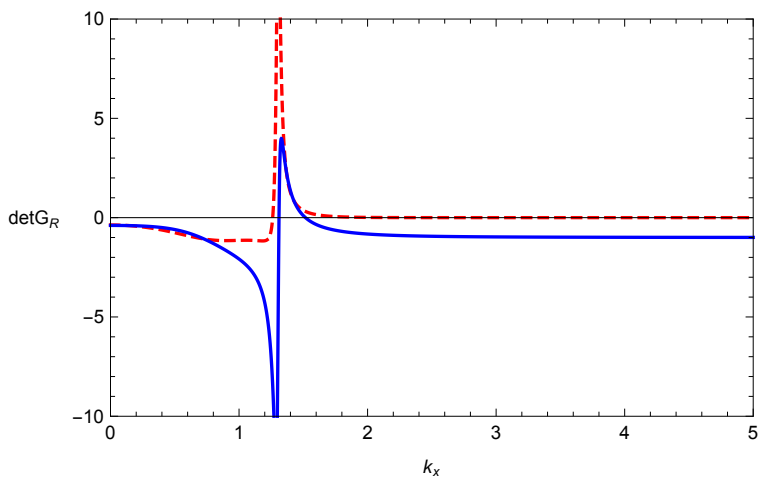

(a)

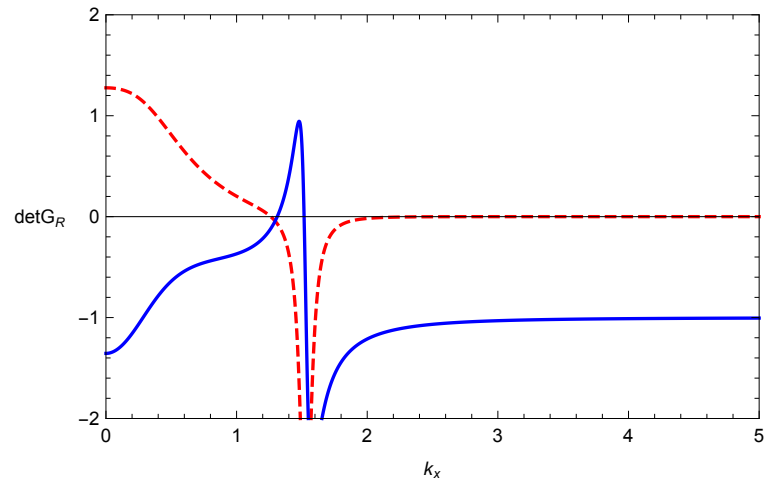

(b)

FIG. 5: $\operatorname{Re}\left(\operatorname{det} G_{R}\right)$ (solid blue line) and $\operatorname{Im}\left(\operatorname{det} G_{R}\right)$ (dashed red line) as a function of $k_{x}$ (we set $\left.k_{y}=0\right)$ at $\omega=0$ with $p=-0.1((\mathrm{a}))$ and $p=0.1((\mathrm{~b}))$ on anisotropic Q-lattice background with $\lambda_{1}=1, \lambda_{2}=0.1$ and $k_{1}=k_{2}=0.8$. Both pole and zero can be seen for $p=-0.1\left(k_{x}=k_{x L} \simeq 1.479\right.$ and $\left.k_{x}=k_{x F} \simeq 1.329\right)$ and $p=0.1\left(k_{x}=k_{x L} \simeq 1.329\right.$ and $\left.k_{x}=k_{x F} \simeq 1.479\right)$.

dipole coupling. Therefore, varying the dipole coupling parameter $p$ can induce quantum phase transitions in our holographic system. By investigating the spectral function, we can work out the critical value $p_{c}$ of the phase transition from (non-)Fermi liquid to Mott phase and see how the lattice parameters $\lambda_{1,2}$ and $k_{1,2}$ affect the formation of the Mott phase in [35]. Here, we have further computed the determinant of the retarded Green function and focused on the formation of the pseudo-gap phase. We find that the region of the pseudo-gap 
phase is suppressed in the deep insulating phase. For the anisotropic Q-lattice geometry, we have obtained an anisotropic pseudo-gap phase region which is also suppressed along the insulating direction. Another interesting result is that the duality between zeros and poles previously found in [10] still holds in either isotropic or anisotropic Q-lattice geometry and is independent of the lattice parameters.

\section{Acknowledgments}

We are grateful to Xiao-Mei Kuang for valuable discussions. This work is supported by the Natural Science Foundation of China under Grant Nos.11275208, 11305018 and 11178002. Y.L. also acknowledges the support from Jiangxi young scientists (JingGang Star) program and 555 talent project of Jiangxi Province. J. P. Wu is also supported by Program for Liaoning Excellent Talents in University (No. LJQ2014123).

[1] J. M. Maldacena, The large $N$ limit of superconformal field theories and supergravity, Adv. Theor. Math. Phys. 2 (1998) 231 [Int. J. Theor. Phys. 38 (1999) 1113].

[2] S. S. Gubser, I. R. Klebanov and A. M. Polyakov, A semiclassical limit of the gauge string correspondence, Nucl. Phys. B 636 (2002) 99.

[3] E. Witten, Anti-de Sitter space and holography, Adv. Theor. Math. Phys. 2 (1998) 253.

[4] S. S. Lee, A Non-Fermi Liquid from a Charged Black Hole; A Critical Fermi Ball, Phys. Rev. D 79 (2009) 086006, arXiv:0809.3402.

[5] H. Liu, J. McGreevy and D. Vegh, Non-Fermi liquids from holography, Phys. Rev. D 83 (2011) 065029 arXiv:0903.2477].

[6] T. Faulkner, H. Liu, J. McGreevy and D. Vegh, Emergent quantum criticality, Fermi surfaces and $A d S_{2}$, Phys. Rev. D 83 (2011) 125002, arXiv:0907.2694.

[7] M. Cubrovic, J. Zaanen and K. Schalm, String Theory, Quantum Phase Transitions and the Emergent Fermi-Liquid, Science 325 (2009) 439 arXiv:0904.1993.

[8] M. Edalati, R. G. Leigh, P. W. Phillips, Dynamically Generated Gap from Holography: Mottness from a Black Hole, Phys.Rev.Lett. 106 (2011) 091602, arXiv:1010.3238.

[9] M. Edalati, R. G. Leigh, K. W. Lo, P. W. Phillips, Dynamical Gap and Cuprate-like Physics 
from Holography, Phys.Rev. D83 (2011) 046012, arXiv:1012.3751.

[10] J. Alsup, E. Papantonopoulos, G. Siopsis, and K. Yeter, Duality between zeroes and poles in holographic systems with massless fermions and a dipole coupling, arXiv:1404.4010.

[11] G. Vanacore, P. W. Phillips, Minding the Gap in Holographic Models of Interacting Fermions, Phys. Rev. D 90, 044022 (2014), arXiv:1405.1041].

[12] J. P. Wu, Holographic fermions in charged Gauss-Bonnet black hole, JHEP 1107 (2011) 106, arXiv:1103.3982.

[13] J. P. Wu, Some properties of the holographic fermions in an extremal charged dilatonic black hole, Phys. Rev. D 84 (2011) 064008, arXiv:1108.6134.

[14] W. J. Li, J. P. Wu, Holographic fermions in charged dilaton black branes, Nucl. Phys. B 867 (2013) 810-826, arXiv:1203.0674.

[15] N. Iizuka, N. Kundu, P. Narayan, S. P. Trivedi, Holographic Fermi and Non-Fermi Liquids with Transitions in Dilaton Gravity, JHEP 1201 (2012) 094, arXiv:1105.1162.

[16] J. P. Wu, Holographic fermions on a charged Lifshitz background from Einstein-DilatonMaxwell model, JHEP 1303 (2013) 083.

[17] X. M. Kuang, E. Papantonopoulos, B. Wang and J. P. Wu, Formation of Fermi surfaces and the appearance of liquid phases in holographic theories with hyperscaling violation, JHEP 1411 (2014) 086 arXiv:1409.2945.

[18] L. Q. Fang, X. H. Ge, X. M. Kuang, Holographic fermions in charged Lifshitz theory, Phys. Rev. D 86 (2012) 105037, arXiv:1201.3832.

[19] L. Q. Fang, X. H. Ge, X. M. Kuang, Holographic fermions with running chemical potential and dipole coupling, Nucl.Phys. B 877 (2013) 807-824, arXiv:1304.7431.

[20] M. Alishahiha, M. Reza Mohammadi Mozaffar, A. Mollabashi, Fermions on Lifshitz Background, Phys. Rev. D 86 (2012) 026002 arXiv:1201.1764].

[21] D. Maity, S. Sarkar, B. Sathiapalan, R. Shankar and N. Sircar, Properties of CFTs dual to Charged BTZ black-hole, Nucl. Phys. B 839 (2010) 526-551, arXiv:0909.4051.

[22] J. P. Wu, H. B. Zeng, Dynamic gap from holographic fermions in charged dilaton black branes, JHEP 1204 (2012) 068, arXiv:1201.2485.

[23] J. P. Wu, Emergence of gap from holographic fermions on charged Lifshitz background, JHEP $04(2013) 073$.

[24] J. P. Wu, The charged Lifshitz black brane geometry and the bulk dipole coupling, Phys. Lett. 
B 728 (2014) 450-456.

[25] X. M. Kuang, B. Wang, J. P. Wu, Dipole coupling effect of holographic fermion in the background of charged Gauss-Bonnet AdS black hole, JHEP 07 (2012) 125, arXiv:1205.6674.

[26] X. M. Kuang, B. Wang, J. P. Wu, Dynamical gap from holography in the charged dilaton black hole, Class. Quant. Grav. 30 (2013) 145011, arXiv:1210.5735.

[27] L. Q. Fang, X. H. Ge, X. M. Kuang, Holographic fermions with running chemical potential and dipole coupling, Nucl. Phys. B 877 (2013) 807-824, arXiv:1304.7431.

[28] W. J. Li, H. Zhang, Holographic non-relativistic fermionic fixed point and bulk dipole coupling, JHEP 1111 (2011) 018, arXiv:1110.4559.

[29] S. R. Coleman, Q balls, Nucl. Phys. B 262 (1985) 263.

[30] A. Donos, J. P. Gauntlett, Holographic Q-lattices, JHEP 1404 (2014) 040, arXiv:1311.3292.

[31] A. Donos, J. P. Gauntlett, Novel metals and insulators from holography, JHEP 1406 (2014) 007.

[32] M. Blake, A. Donos, Quantum Critical Transport and the Hall Angle, arXiv:1406.1659.

[33] A. Donos, J. P. Gauntlett, Thermoelectric DC conductivities from black hole horizons, arXiv:1406.4742.

[34] Y. Ling, P. Liu, C. Niu, J. P. Wu, and Z. Y. Xian, Holographic Superconductor on Q-lattice, JHEP 02 (2015) 059, arXiv:1410.6761.

[35] Y. Ling, P. Liu, C. Niu, J. P. Wu, and Z. Y. Xian, Holographic fermionic system with dipole coupling on Q-lattice, JHEP 12 (2014) 149, arXiv:1410.7323.

[36] Y. Ling, P. Liu, C. Niu, J. P. Wu, and Z. Y. Xian, Holographic Entanglement Entropy Close to Quantum Phase Transitions, arXiv:1502.03661].

[37] Y. Ling, P. Liu, C. Niu, J. P. Wu, Building a doped Mott system by holography, arXiv:1507.02514.

[38] T. D. Stanescu, P. W. Phillips, and T. Choy, P Theory of the Luttinger surface in doped Mott insulators, hys. Rev. B 75, 104503 (2007), arXiv:cond-mat/0602280].

[39] I. Dzyaloshinskii, Some consequences of the Luttinger theorem: The Luttinger surfaces in non-Fermi liquids and Mott insulators, Phys. Rev. B 68, 085113 (2003).

[40] S. Hong and P. W. Phillips, Towards the standard model for Fermi arcs from a Wilsonian reduction of the Hubbard model, Phys. Rev. B 86, 115118 (2012), arXiv:1110.0440 [condmat.str-el]]. 
[41] S. Sakai, Y. Motome, and M. Imada, Evolution of Electronic Structure of Doped Mott Insulators: Reconstruction of Poles and Zeros of Green's Function, Phys. Rev. Lett. 102, 056404 (2009), arXiv:0809.0950v1 [cond-mat.str-el]].

[42] S. Sakai, Y. Motome, and M. Imada, Doped high-Tc cuprate superconductors elucidated in the light of zeros and poles of the electronic Green's function, Phys. Rev. B 82, 134505 (2010), arXiv:1004.2569 [cond-mat.str-el]]. 